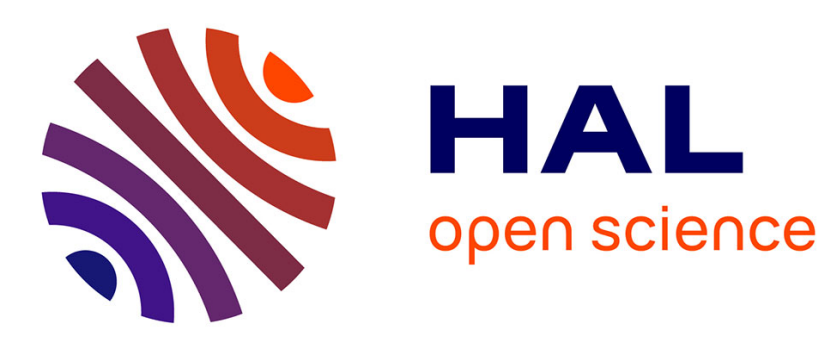

\title{
Ingestion spontanée d'aliment chez la truie en lactation : de nombreux facteurs de variation
}

Jean-Yves Dourmad

\section{To cite this version:}

Jean-Yves Dourmad. Ingestion spontanée d'aliment chez la truie en lactation: de nombreux facteurs de variation. Productions Animales, 1988, 1 (2), pp.141-146. hal-00895826

\section{HAL Id: hal-00895826 \\ https://hal.science/hal-00895826}

Submitted on 1 Jan 1988

HAL is a multi-disciplinary open access archive for the deposit and dissemination of scientific research documents, whether they are published or not. The documents may come from teaching and research institutions in France or abroad, or from public or private research centers.
L'archive ouverte pluridisciplinaire HAL, est destinée au dépôt et à la diffusion de documents scientifiques de niveau recherche, publiés ou non, émanant des établissements d'enseignement et de recherche français ou étrangers, des laboratoires publics ou privés. 
INRA Prod. Anim., 1988, 1 (2), 141-146

\section{J.Y. DOURMAD}

INRA Saint-Gilles

Station de Recherches Porcines

35590 L'Hermitage

\section{Ingestion spontanée d'aliment chez la truie en lactation : de nombreux facteurs de variation}

\begin{abstract}
Les besoins nutritionnels de la truie en lactation sont particulièrement élevés, aussi le niveau d'ingestion spontané d'aliment est-il généralement insuffisant pour en assurer la couverture. L'étude des différents facteurs de variation de la consommation spontanée doit permettre d'optimiser les quantités d'aliment ingérées par la truie en lactation et ainsi de couvrir le mieux possible les besoins.
\end{abstract}

Les besoins nutritionnels de la truie en lactation sont d'autant plus importants que le niveau de production laitière et, par conséquent, la croissance de la portée sont élevés. Ainsi, une truie de $180 \mathrm{~kg}$ produisant en moyenne $8 \mathrm{~kg}$ de lait par jour devra consommer quotidiennement, pour couvrir ses besoins en énergie, plus de $6 \mathrm{~kg}$ d'un aliment contenant $3100 \mathrm{kcal}$ d'énergie digestible (ED) par kg. L'ingestion alimentaire spontanée en lactation permet de satisfaire ces besoins seulement chez les truies faibles productrices, les autres étant systématiquement en déficit énergétique. Lorsque les réserves corporelles de la truie sont suffisantes, ce déficit n'entraîne pas de réduction de la production laitière, ni de la croissance des porcelets (Noblet et Etienne 1987). Par contre, si cette situation se reproduit pendant plusieurs cycles successifs, on observe un effet négatif sur la production de lait et une réduction de la croissance des porcelets (O'Grady et al 1973). Dans tous les cas, ce déficit s'accompagne d'une mobilisation importante des réserves adipeuses et musculaires (Duée et Desmoulin 1982, Noblet et Etienne 1987) qui peut affecter les performances de reproduction à moyen et à long terme, en particulier l'intervalle sevrage-oestrus (Reese $e t$ al 1984, King et Williams 1984).

Aussi une alimentation à volonté des truies allaitant plus de dix porcelets est-elle généralement recommandée (INRA 1984) afin de minimiser les variations de l'état des réserves corporelles au cours des cycles successifs. Cette revue bibliographique a pour objectif de présenter les différents facteurs liés à l'animal, à l'aliment ou aux conditions d'élevage qui peuvent influencer le niveau d'ingestion spontanée chez la truie en lactation.

\section{1 / Facteurs liés à l'animal}

\section{Résumé}

Chez la truie allaitante, le niveau spontané d'ingestion alimentaire est généralement trop faible pour couvrir les besoins nutritionnels élevés liés à la production de lait. Ceci se traduit par une mobilisation des réserves corporelles qui est néfaste aux performances de reproduction ultérieures.

Lanalyse de la bibliographie montre que de nombreux facteurs peuvent affecter le niveau de consommation en lactation. Il augmente avec le stade de lactation, le numéro de portée et la taille de la portée. L'élévation de la teneur en énergie du régime entraîne une diminution de la quantité consommée, mais malgré cela l'ingéré énergétique est supérieur. D'autre part, lorsque la teneur en protéines est inférieure à $12 \%$, la quantité consommée diminue. Laugmentation des apports alimentaires en gestation entraîne une réduction de l'appétit en lactation, ce résultat étant à rapprocher de l'effet négatif d'un état d'engraissement trop élevé à la mise-bas. Enfin, les températures ambiantes élevées en maternité se traduisent par une réduction importante de la consommation. Ces différents effets peuvent se cumuler. Aussi certains animaux sont particulièrement prédisposés à des problèmes de sousconsommation en lactation, par exemple les truies primipares mettant bas en été et ayant reçu une alimentation libérale au cours de la gestation précédente.

\section{1 / Stade de la lactation}

La capacité d'ingestion de la truie augmente au fur et à mesure de l'avancement de la lactation (figure 1, NRC 1987), les variations étant surtout importantes au cours de la première semaine. Par la suite, l'ingestion alimentaire se stabilise à un niveau maximum qui semble se maintenir au moins jusqu'à la quatrième semaine de lactation. En raison du faible nombre de résultats disponibles, il est difficile de préciser l'évolution ultérieure de la consommation pour des lactations plus longues.

L'augmentation de l'ingestion spontanée avec le stade de lactation est similaire à l'évolution de la production laitière, dont le maximum est atteint au cours de la troisième semaine. Ceci semble montrer une influence possible du niveau de production sur le niveau de consommation. 
Figure 1. Evolution du niveau d'ingestion spontanée en fonction du stade de lactation (d'après NRC, 1987).



Les lactations courtes se traduisent donc par un niveau d'ingestion moyen journalier plus faible. La quantité d'aliment reçue au cours de la première semaine de lactation ne semble pas influencer l'appétit au cours des semaines suivantes. Ainsi, après 7 jours de lactation, Stahly et al (1979) n'observent pas de différence d'ingestion journalière d'aliment entre les truies placées ad libitum dès la mise-bas et celles rationnées suivant une échelle croissante d'alimentation au cours de la première semaine de lactation.

\section{2 / Taille de la portée}

La production laitière s'accroît de façon importante avec le nombre de porcelets allaités. Elle est doublée lorsque la taille de la portée passe de quatre à onze porcelets. La couverture des besoins supplémentaires liés à l'augmentation de la production de lait se fait grâce à la mobilisation de réserves corporelles, mais également par une élévation du niveau d'ingestion spontanée. O'Grady et al (1985) ont observé, sur des résultats d'élevage, une relation non linéaire entre le nombre de porcelets sevrés et le niveau de consommation de la truie. Comme le montre la figure 2 , l'augmentation moyenne de l'ingestion par porcelet supplémentaire est d'autant plus importante que la taille de la portée est faible. Ainsi, lorsque le nombre de porcelets sevrés passe de 8 à 12 , le niveau de consommation de la mère augmente seulement de $250 \mathrm{~g} /$ jour. Ceci est notoirement insuffisant pour satisfaire les besoins énergétiques supplémentaires, puisque ces derniers s'élèvent à plus de $1,5 \mathrm{~kg} / \mathrm{jour}$.

\section{3 / Numéro de portée}

Le numéro de portée affecte significativement l'ingestion spontanée d'aliment chez la truie en lactation (tableau 1). En moyenne la consommation est supérieure d'environ $13 \%$ en seconde portée par rapport à la première. Selon O'Grady et al (1985), l'augmentation est importante entre la première et la seconde portée puis se poursuit à un niveau moindre jusqu'à la quatrième. Cette évolution de la consommation avec le numéro de portée s'explique en partie par l'augmentation concomitante du poids de la truie et de la taille de la portée.

\section{4 / Type génétique}

Dans une synthèse bibliographique sur la régulation de l'appétit chez le porc à l'engrais, Henry (1985) concluait à un niveau d'ingestion spontanée plus faible chez les souches de porcs de type "maigre" que chez les souches de type "gras". Cet effet génétique se retrouve chez la truie en lactation mais, semble-t-il, de façon moins marquée. Les truies de race Large White consomment $15 \%$ d'aliment de plus que les truies croisées Hampshire $\times$ Chester White, plus maigres (Pond et al 1981). De même O'Grady et al (1985) constatent une consommation plus élevée chez les truies (2/3 Large White $x$ $1 / 3$ Landrace) que chez les truies ( $2 / 3$ Landrace $\times 1 / 3$ Large White). On peut donc se demander si les sché-

Figure 2. Relation entre le niveau d'ingestion spontanée de la truie en lactation et le nombre de porcelets sevrés (d'après O'Grady et al 1985).

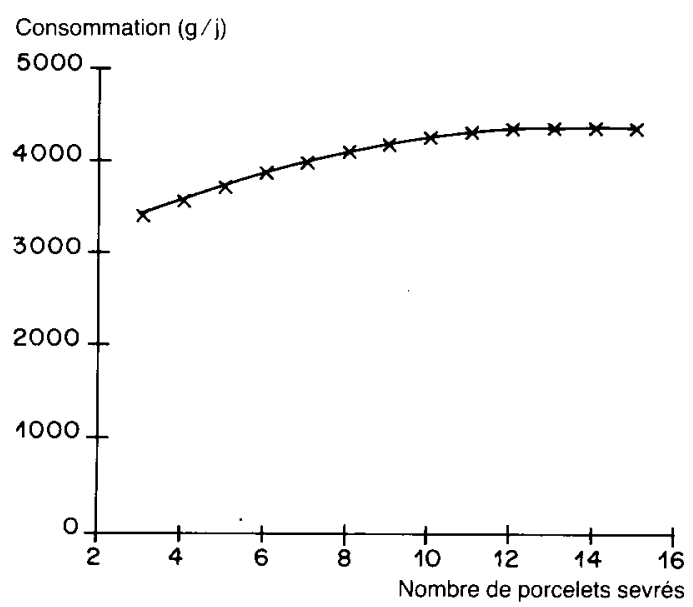

Tableau 1. Influence du numéro de portée sur l'ingestion spontanée chez la truie en lactation. Analyse statistique de 8 résultats bibliographiques.

\begin{tabular}{|lccc|c|}
\hline Numéro de portée & $\mathbf{1}$ & 2 & 3 & $\begin{array}{c}\text { Signification } \\
\text { statistique (1) }\end{array}$ \\
\hline Consommation journalière & & & & $*$ \\
$\quad$ - en kg / jour & 4,74 & 5,33 & 5,11 & NS \\
$\quad$ en g / kg poids vif & 27,8 & 28,4 & 24,9 & $* *$ \\
Porcelets nés & 9,8 & 10,2 & 10,7 & $* *$ \\
Poids de la truie $(\mathrm{kg})$ & 171 & 192 & 209 & \\
\hline
\end{tabular}

(1) *P<0,05;** $\mathrm{P}<0,01$; NS Non significatif. 
Tableau 2. Relation entre le poids de la truie après la mise-bas et le niveau de consommation spontané en lactation (d'après O'Grady et al 1985).

\begin{tabular}{|c|c|c|c|c|c|c|c|}
\hline & \multicolumn{6}{|c|}{ Poids vif après la mise-bas $(\mathrm{kg})$} & \multirow{2}{*}{$\begin{array}{c}\text { Signification } \\
\text { statistique } \\
\text { (1) }\end{array}$} \\
\hline & $\begin{array}{c}\text { inférieur } \\
\text { à } 140\end{array}$ & $\begin{array}{c}141 \text { à } \\
160\end{array}$ & $\begin{array}{c}161 \text { à } \\
180\end{array}$ & $\begin{array}{c}181 \text { à } \\
200\end{array}$ & $\begin{array}{c}201 \text { à } \\
220\end{array}$ & $\begin{array}{l}\text { supérieur } \\
\text { à } 220\end{array}$ & \\
\hline Effectif des truies & 138 & 362 & 517 & 719 & 748 & 446 & - \\
\hline $\begin{array}{l}\text { Aliment consommé } \\
\mathrm{kg} / \mathrm{j}\end{array}$ & 3,93 & 4,19 & 4,52 & 4,73 & 4,82 & 4,71 & $* *$ \\
\hline $\begin{array}{l}\text { Besoin d'entretien } \\
\text { kcal EM / j (2) }\end{array}$ & - & 4930 & 5410 & 5885 & 6345 & - & - \\
\hline $\begin{array}{l}\text { Energie disponible } \\
\text { kcal EM / j }\end{array}$ & - & 7640 & 8150 & 8305 & 8115 & - & - \\
\hline
\end{tabular}

(1) * P $<0,05 ; * * P<0,01 ;-$ Non testé ; NS Non significatif

(2) $115 \mathrm{kcal} \mathrm{EM} / \mathrm{kg}^{0,75}$

Figure 3. Fréquence de l'anorexie, chez la truie, au cours des cinq jours suivant la mise-bas (d'après Madec 1983).

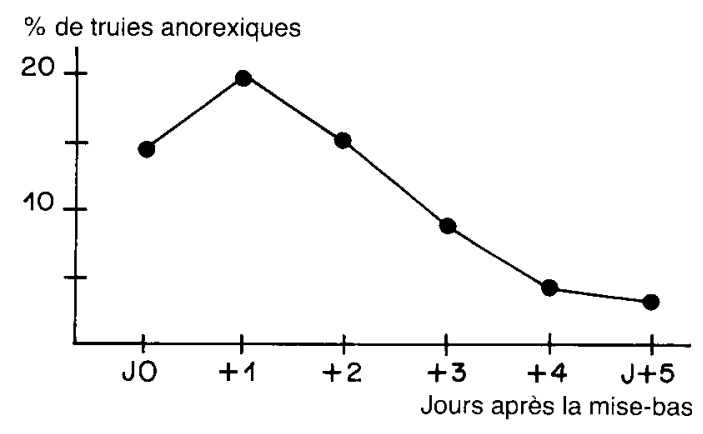

mas de sélection actuels, dont l'objectif principal est l'amélioration de la teneur en muscle de la carcasse, n'entraînent pas une réduction de l'appétit chez la truie.

\section{5 / Poids et état d'engraissement de la truie}

Laugmentation de poids de la truie s'accompagne jusqu'à environ $200 \mathrm{~kg}$ d'un accroissement du niveau $\mathrm{d}$ 'ingestion légèrement supérieur aux variations $\mathrm{du}$ besoin énergétique d'entretien (tableau 2).

D'autre part, pour un type génétique donné, l'appétit tend à diminuer chez les truies très grasses, alors qu'il s'accroît chez les truies maigres (O'Grady et al 1985). Ceci semble mettre en évidence le rôle des réserves (adipeuses et/ou musculaires) dans les mécanismes de régulation de l'appétit, alors que l'effet du type génétique serait le résultat d'une sélection à long terme sur un critère lié à l'appétit.

\section{6 / Etat sanitaire}

La fréquence d'apparition d'anorexie totale est maximale le jour suivant la parturition (Madec 1983, figure 3). Ce phénomène ne peut pas être attribué entièrement à des problèmes d'ordre pathologique. Cependant certaines affections se traduisent par une réduction de l'appétit, c'est le cas des cystites et métrites, ou d'anorexie totale dans le cas de mammites. D'autre part, certaines affections telles que néphrites et pneumonies enzootiques présentent une recrudescence en lactation et entraînent, entre autres, des pertes d'appétit (Vannier et al 1983).

\section{2 / Facteurs liés au milieu}

\section{$2.1 /$ Température}

La température ambiante du bâtiment influence notablement le niveau d'ingestion chez la truie en lactation. Lynch (1978, tableau 3) observe une augmentation de la consommation journalière de $30 \%$ chez les truies placées à une température de $16^{\circ} \mathrm{C}$ par rapport à celles se trouvant à $27^{\circ} \mathrm{C}$, soit un écart de plus de $120 \mathrm{~g} /{ }^{\circ} \mathrm{C} /$ jour ; entre 27 et $21^{\circ} \mathrm{C}$ l'écart observé est de $100 \mathrm{~g} /{ }^{\circ} \mathrm{C} /$ jour. Dans cette expérience l'augmentation de consommation qui résulte de la baisse de la température ambiante se traduit également par une diminution de la perte de poids de la mère et une augmentation du poids moyen des porcelets au sevrage. Dans une étude plus ancienne Jensen (1964) obtient des résultats analogues, l'effet étant cependant légèrement plus faible : $80 \mathrm{~g} /{ }^{\circ} \mathrm{C} /$ jour entre 18 et $28^{\circ} \mathrm{C}$.

Durant la lactation il faut donc pouvoir satisfaire les besoins élevés en température des porcelets (plus de $25^{\circ} \mathrm{C}$ ) tout en évitant une température trop forte pour la truie, ce qui conduit à préconiser l'usage de nids à porcelets.
Température ambiante $\left({ }^{\circ} \mathrm{C}\right)$ Consommation $(\mathrm{kg} / \mathrm{j})$ Perte de poids de la truie $(\mathrm{kg})$ Nombre de porcelets sevrés Poids moyen au sevrage $(\mathrm{kg})$

\begin{tabular}{|cc|cc|}
\hline \multicolumn{2}{|c|}{ Expérience 1 } & \multicolumn{2}{c|}{ Expérience 2 } \\
\hline 27 & 21 & 27 & 16 \\
4,6 & 5,2 & 4,2 & 5,6 \\
21 & 14 & 22 & 13 \\
8,1 & 7,9 & 8,0 & 8,0 \\
6,2 & 7,0 & 6,4 & 7,3 \\
\hline
\end{tabular}

Tableau 3. Influence de la température ambiante sur la consommation spontanée chez la truie et les performances de la portée (d'après Lynch 1978). 


\section{2 / Lumière}

Des études récentes montrent une influence favorable de l'intensité et de la durée d'éclairement sur la croissance des porcelets et la production laitère de la mère (Mabry et al 1983, Stevenson et al 1983). A notre connaissance les mécanismes impliqués dans ce phénomène n'ont pas été étudiés chez la truie. L'hypothèse d'une action de la lumière sur la consommation d'aliment a été avancée (Mabry et al 1983), sans cependant savoir s'il s'agit d'un effet direct ou simplement une réponse à l'augmentation de la production laitière.

\section{3 / Facteurs liés à l'aliment}

\section{1 / Teneur en énergie de l'aliment}

Le niveau d'ingestion spontané chez la truie en lactation tend à diminuer lorsque la teneur en énergie du régime augmente (Figure 4). On peut estimer que pour $100 \mathrm{kcal}$ d'augmentation de la teneur de l'aliment en énergie métabolisable le niveau d'ingestion diminue de $64 \mathrm{~g} /$ jour. Dans une gamme classique de concentrations énergétiques le niveau d'ingéré énergétique reste cependant en faveur des régimes les plus concentrés. Ainsi pour une augmentation de $17 \%$ de la teneur en énergie métabolisable (3000 à $3500 \mathrm{kcal} / \mathrm{kg})$, on peut estimer la baisse de consommation d'aliment à $7 \%$ alors que la quantité totale d'énergie ingérée s'accroît de $9 \%$. Cependant, la nature de l'énergie apportée peut modifier la réponse en terme de bilan énergétique pour la truie. En effet, si l'on augmente la teneur en énergie du régime en le supplémentant en matière grasses on augmente également l'exportation de lipides et donc d'énergie dans le lait ; et dans ce cas, le bilan énergétique de la truie n'est pas meilleur (Dourmad 1987).

Comme cela a été montré chez le porc à l'engrais, il semble exister chez la truie en lactation un interaction entre la température ambiante et la concentration énergétique du régime. Ainsi à une température élevée $\left(21,5^{\circ} \mathrm{C}\right)$ O'Grady et Lynch (1978) n'observent plus d'influence de la concentration en énergie sur la consommation en $\mathrm{kg} / \mathrm{j}$. L'augmentation de la teneur en énergie de l'aliment a de ce fait souvent été recommandée pour pallier l'effet dépressif de températures élevées sur le niveau d'ingestion spontanée d'énergie.
Figure 4. Relation entre la concentration énergétique du régime et le niveau d'ingestion spontané chez la truie en lactation (synthèse de résultats bibliographiques).

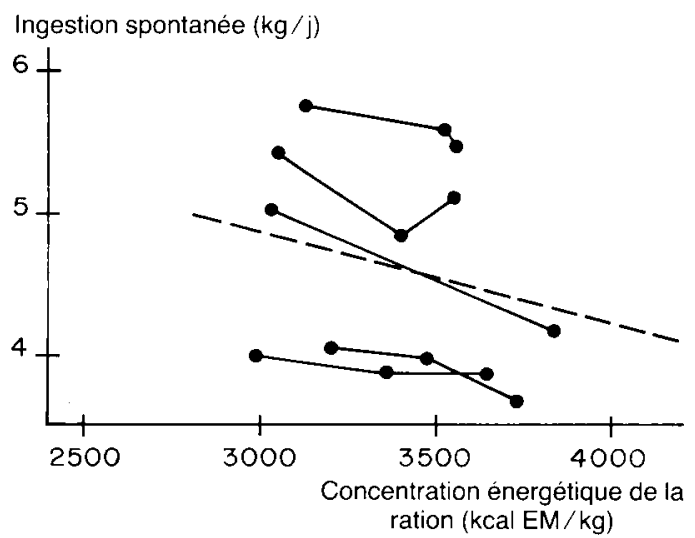

\section{2 / Teneur en protéines et en acides aminés}

Le taux de protéines affecte significativement le niveau d'ingestion alimentaire spontanée en lactation (tableau 4). Lorsque ce taux est inférieur à $12 \%$, on observe une réduction systématique de la consommation (de $15 \%$ en moyenne) par rapport aux régimes à $16 \%$ de protéines. Par contre aucun effet similaire n'est observé au-dessus de 14-15\% de protéines.

De façon générale le taux azoté du régime de lactation n'influence donc le niveau d'ingestion qu'en-dessous de $12 \%$ de protéines dans le régime, cet effet étant d'autant plus marqué que l'apport protéique en gestation est faible (Mahan et Mangan 1975).

En ce qui concerne la composition du régime en acides aminés, Lewis et Speer (1975) observent une réduction spectaculaire de l'ingestion lorsque la teneur en tryptophane du régime est inférieure à $0,07 \%$. Cet effet d'une carence en tryptophane est classique dans l'espèce porcine. Il est donc particulièrement important de couvrir les besoins qui sont estimés à $0,13 \%$. Par contre pour la lysine, qui est l'acide aminé limitant primaire des régimes pour les porcs, aucun effet sur l'ingestion n'est observé pour des teneurs variant entre 0,6 et $1 \%$ (Boomgart et al 1972).

Tableau 4. Influence du taux protéique du régime sur le niveau d'ingestion à volonté durant la lactation (1).

\begin{tabular}{|l|c|c|c|}
\hline \multirow{2}{*}{} & \multicolumn{3}{|c|}{ Teneur en protéines (\% du régime) } \\
\cline { 2 - 4 } & $\begin{array}{c}\text { inférieure } \\
\text { à } 12 \%\end{array}$ & $\begin{array}{c}\text { entre } 14 \% \\
\mathbf{1 4} \% \text { et } 15 \%\end{array}$ & $\begin{array}{c}\text { supérieure } \\
\text { à } 16 \%\end{array}$ \\
\hline Mahan et Mangan 1975 & - & $\ldots$ & $=$ \\
Mahan et Grifo 1975 & - & $=$ & $=$ \\
Mahan et Grifo 1975 & $\ldots$ & $=$ & $=$ \\
Mahan 1977 & $\ldots$ & $=$ & $=$ \\
Shields et al 1985 & - & $\ldots$ & $=$ \\
King et William 1984 & - & $\ldots$ & $=$ \\
O'Grady 1971 & - & & \\
\hline
\end{tabular}

(1) - Consommation significativement inférieure $\mathrm{a}=$ et $\mathrm{a}+$

+ Consommation significativement supérieure à $=$ et à -

... Domaine non couvert par l'expérience 


\section{3 / Autres facteurs liés à l'aliment}

Lingestion d'aliment peut, comme chez le porcelet ou le porc en croissance, être réduite par la présence de substances inappétentes ou toxiques. Cette situation 'est souvent la conséquence d'une mauvaise conservation des matières premières ou de l'aliment complet luimême. L'intoxication chronique par les trichothécènes, mycotoxine produite par plusieurs moisissures, se traduit ainsi par une réduction de l'appétit, l'intoxication aiguë entraînant un refus total de l'aliment (Vannier $e t$ al 1982).

L'introduction à taux élevé (20\%) de tourteau de colza simple $O$ peut entraîner des sous-consommations passagères qui disparaissent après quelques jours (Etienne et Dourmad 1987).

\section{4 / Facteurs liés à la conduite d'élevage et aux techniques d'alimentation}

\section{1 / Influence du niveau alimentaire et du gain de poids au cours de la gestation précédente}

Les pertes pondérales en lactation sont d'autant plus importantes que le niveau alimentaire et le gain de poids au cours de la gestation précédente sont élevés (Henry



Figure 5. Influence du niveau énergétique journalier de gestation sur la consommation spontanée au cours de la lactation suivante (synthèse de résultats bibliographiques). et Etienne 1978). Par ailleurs, une alimentation libérale en gestation se traduit généralement par une réduction de l'appétit en lactation (Salmon-Legagneur 1969, Baker et al 1968, Buitrago et al 1974, Brooks 1982, Burlacu et al 1983). On peut évaluer à 170 g/jour en moyenne, la réduction de consommation spontanée en lactation consécutive à un accroissement du niveau alimentaire de gestation de $1000 \mathrm{kcal} \mathrm{ED} / \mathrm{j}$ (figure 5). Ces résultats demandent cependant à être réévalués pour des lactations plus courtes, particulièrement chez la truie primipare où l'effet semble être accentué

Par contre, une suralimentation limitée à la fin de la gestation n'a pas en général d'effet dépressif significatif sur l'appétit en lactation (Pond et al 1981, Cromwell et al 1982, Walker et al 1982).

Tableau 5. Récapitulatif des principaux facteurs de variation de l'ingestion alimentaire spontanée chez la truie en lactation.

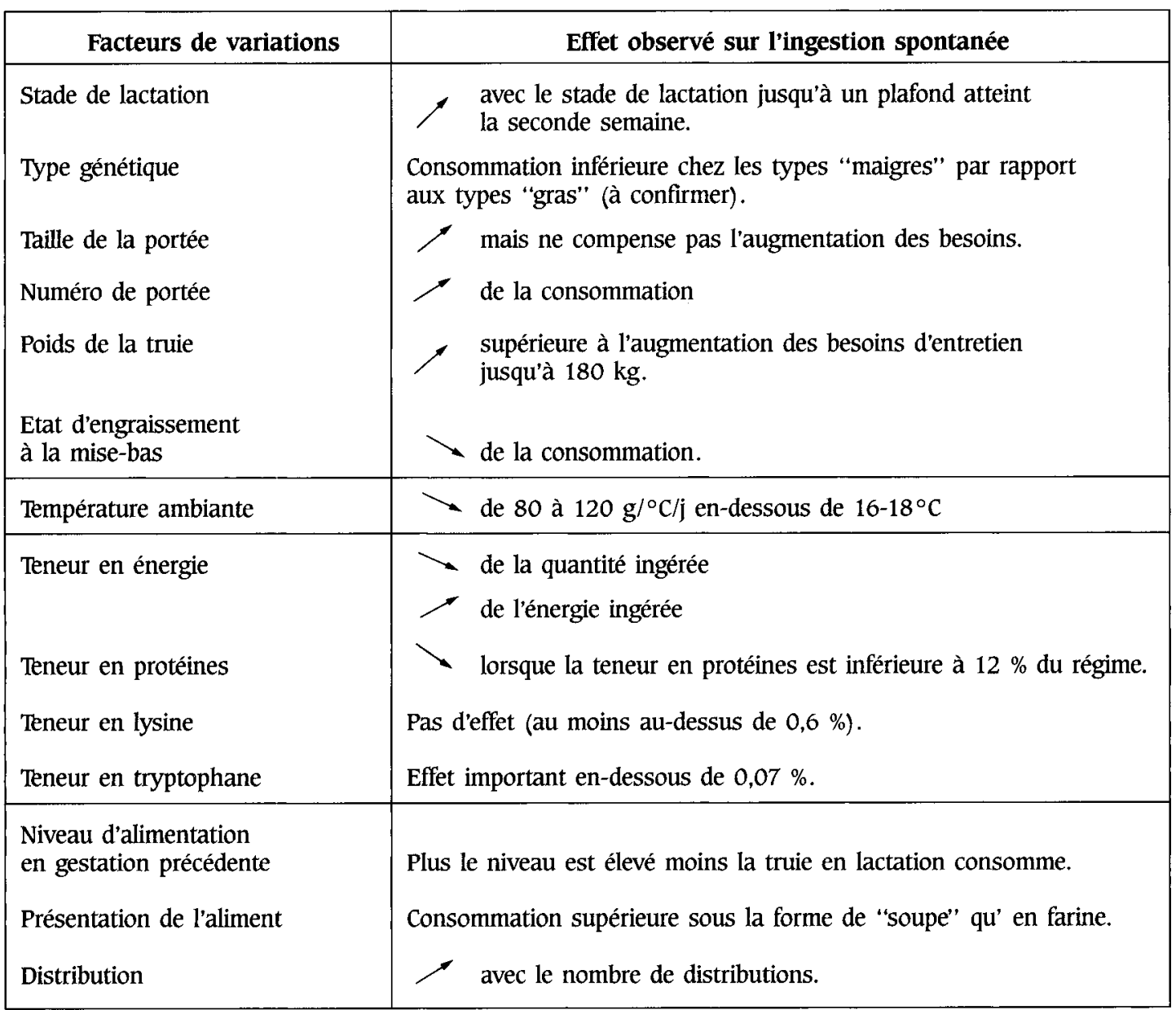

Une alimentation libérale en gestation se traduit généralement par une réduction de l'appétit en lactation. 


\section{2 / Techniques d'alimentation}

Le rythme des apports d'aliment influence également le niveau d'ingestion spontané. Libal et Wahlstrom (1983) observent ainsi, en alimentation à volonté, que la consommation augmente de $15 \%$ lorsque l'aliment est distribué 3 fois par jour, au lieu de une seule. On peut penser que cet effet serait lié à une influence sur le comportement de la truie, les animaux se levant plus souvent.

La présentation de l'aliment sous la forme de soupe accroît le niveau d'ingestion d'environ $12 \%$ (O'Grady et Lynch 1978, Danielsen et Nielsen 1984).

\section{Conclusion}

L'ingestion spontanée d'aliment chez la truie en lactation est donc influencée par un ensemble de facteurs liés à l'aliment, à la conduite d'élevage et à l'animal luimême. Dans le tableau 5 sont rappelés les principaux effets étudiés. Il est parfois difficile de quantifier les effets respectifs de ces différents facteurs, d'autant plus que des interactions semblent exister entre eux. Cependant certains animaux sont particulièrement prédisposés aux problèmes de sous-consommation en lactation, c'est par exemple le cas des truies primipares mettant bas en période estivale et ayant reçu une alimentation libérale en gestation.

Ces différents résultats mettent par ailleurs en évidence la nécessité de tenir compte du niveau d'ingestion spontanée d'aliment en lactation, dans la stratégie d'alimentation sur la totalité du cycle, en modulant éventuellement les apports au cours de la gestation suivante. D'autre part, afin de couvrir les besoins journaliers en protéines et en acides aminés, il est important de tenir compte de la capacité d'ingestion réelle des animaux pour le calcul des teneurs en nutriments des régimes.

\section{Références bibliographiques}

BAKER D.H., BECKER D.E., NORTON H.W., SASSE C.E., JENSEN A.H., HARMON B.G., 1968. Reproductive performance and progeny development in swine as influenced by food intake during pregnancy, J. Nutrition, 97, 489-495.

BOOMGAARDT J., BAKER D.H., JENSEN A.H., HARMON B.G., 1972. Effect of dietary lysine levels on 21-day lactation performance of fist litter sows. J. Anim. Sci., 34, 408-410.

BROOKS P.H., 1982. Feeding the reproducing female. Proceedings, Pig veterinary society 9, 84-102.

BUITRAGO J.Al., MANER J.H., GALLO J.T., POND W.G., 1974. Effet of dietary energy in gestation on reproductive performance of gilts. J. Anim. Sci., 39, 47-52.

BURLACU G.H., ILIESCU Monica, CARAMIDA P., 1983. Efficiency of food with different concentrations of energy on pregnancy and lactation. Arch. Tierernähr., Berlin, 23-45.

CROMWELL G.L., PRINCE T.J., COMBS G.E., MAXWELL C.V., KNABE D.A., ORR D.E., 1982. Effect of additional feed during late gestation on reproductive performance of sows. J. Anim. Sci., 55, Suppl. 1, p. 268 Abs 371

DANIELSEN V., NIELSEN H.E., 1984. The influence of different feeding levels on the performance of lactating sows. 35th Annual Meeting of the E.A.A.P., La Haye 1984.

DOURMAD J.Y., 1987. Intérêt des matières grasses chez la truie. Revue de l'alimentation animale, 405, 39-44.

DUEE P.H., DESMOULIN B., 1982. Modification de la composition corporelle de la truie nullipare au cours d'un cycle de reproduction : effet du taux protéique de gestation. Journées Rech. Por cine en France, 14, 91-96.

ETIENNE M., DOURMAD J.Y., 1987. Effet de la consommation de tourteau de colza normal ou à faible teneur en glucosinoltates sur la reproduction chez la truie. Journées Rech. porcine en France $19,231-238$

HENRY Y., 1985. Dietary factors involved in feed intake regulation in growing pig : a review. Liv. Prod. Sci., 12, 339-354. HENRY Y., ETIENNE M. 1978. Alimentation énergétique du porc In Jour. Rech. Porc. en France, 10, 119-165.

INRA, 1984. L'alimentation des animaux monograstriques. Ed. INRA Paris 67-76.

JENSEN A.H., 1964. Symposium of environment and facilities : Environment and facilities in swine production. J. Anim. Sci, 23, 1185-1196.

KING R.H., WILLIAMS I.H., 1984. The effect of nutrition on the reproductive performance of first litter sows. 1 - Feeding level during lactation, and between weaning and mating. Animal production, 38, 241-247.

LEWIS A.J., SPEER V.C., 1975. Tryptophane requirement of the lactating sow. J. Anim. Sci. 38, 778-784.

LIBAL G.W., WAHLSTROM R.C., 1983. The effect of feeding frequency and feed flavoring on performance of lactating sows and gilts. Proceedings Swine Day South Dakota University (17 nov. 1983) $25-28$.

LYNCH P.B., 1978. Environment and the breeding sow. Proceedings Moorepark pig farming Conference, "An Foras Taluntais", Dublin 18-20.

MABRY J.W., COFFEY M.T., SEERLEY R.W., 1983. A comparison of 8 versus 16 -hour photoperiod during lactation on suckling frequency of the baby pig and maternal performance of the sow. J. Animal Sci., 57, 292-295.

MADEC F., 1983. Troubles de la mise bas : une dizaine de facteurs de risque, l'élevage porcin, août-septembre 1983, 47-53.

MAHAN D.C., 1977. Effect of feeding various gestation and lactation dietary protein sequences on long term reproductive performance in swine. J. Anim. Sci., 45, (5) 1061-1072.

MAHAN D.C., GRIFO A.P. Jr., 1975. Effects of dietary protein levels during lactation to first litter sows fed a fortified corn gestation diet. J. Anim. Sci., 41, 1362-1367.

MAHAN D.C., MANGAN L.T., 1975. Evaluation of various protein sequences on the nutritional carry-over from gestation to lac tation with first-litter sows. J. Nutr., 105, 1291-1298.

NOBLET J., ETIENNE M., 1987. Dépenses et besoins énergétiques de la truie au cours du cycle de reproduction. Journées Rech. porcine en France, 19, 197-202.

NRC, 1987 . In : predicting feed intake of food producing animals 86 pp. Nat. Acad. Press, WASHINGTON.

O'GRADY J.F., 1971. Level and source of protein in the diets of lactating sows. L Ir. J. Agric. Res., 10, 17-29.

O'GRADY J.F., LYNCH P.B., 1978. Voluntary feed intake by lactating sows : Influence of system of feeding and nutrient density of the diet. Ir. J. Agri. Res., 17, 1-5.

O'GRADY J.F., ELSLEY F.W.H., Mac PHERSON R.M., Mac DONALD I., 1973. The response of lactating sows and their litters to different dietary energy allowances. Anim. Prod. 17, 65-74. O'GRADY J.F., LYNCH P.B., KEARNEY P.A., 1985. Voluntary feed intake by lactating sows. Liv. Prod. Sci., 12, 355-365.

POND W.G., YEN J.T., MAURER R.R., CHRISTENSON R.K., 1981. Effect of doubling daily energy intake during the last two weeks of pregnancy on pig birth weight and weaning weight. J. Anim. Sci., 52, $1981535-541$.

REESE D.E., PEO E.R., LEWIS A.J., 1984. Relationship of lactation energy intake and occurence of post weaning oestrus to body and back fat composition in sows. J. Anim. Sci. 58, (5) 1236-1244. SALMON-LEGAGNEUR E., 1969. Influence à long terme du rationnement des truies gestantes. In Journées de la Recherche Porcine en France, 12, 77-81.

SHIELDS, Jr., MAHAN D.C., MAXSON P.F., 1985. Effect of dietary gestation and lactation protein levels on reproductive performance and body composition of first-litter female swine. J. Anim. Sci. $60,179-189$

STAHLY T.S., CROMWELL G.L., SIMPSON W.S., 1979. Effect of Fullvs restricted feeding of sow immediately post partum on lactation performance. J. Anim. Sci., 49 (1), 50-54.

STEVENSON J.S., POLLMAN D.S., DAVIS D.L., MURPHY J.P. 1983. Influence of supplemental light on sow performance during and after lactation. J. Anim. Sci. 56, 1282-1286.

VANNIER P., CONSTANTIN A., NOIRRIT M., DAGORN J., ETIENNE M., RUNAVOT J.P., 1982. Pathologie des reproducteurs, 410-459. In MORNET P., TOURNU J., TOMA B. Le porc et ses maladies. Ed. Maloine S.A. 